\title{
Infinitesimal Discontinuities in Initially Stressed Relativistic Elastic Solids
}

\author{
Gérard A. Maugin \\ Université de Paris VI, Laboratoire de Mécanique Théorique associé au C.N.R.S, \\ F-75230 Paris, France
}

\begin{abstract}
In this paper we examine the influence of a general initial state of stress upon the propagation of infinitesimal (or weak) discontinuities in nonlinear relativistic elasticity. This influence, which materializes in alterations in the wave speeds, the general nonseparability in longitudinal and transverse waves, and the growth of the amplitude of infinitesimal discontinuities so as to form shock fronts, is first studied on the basis of a model of relativistic elasticity called hypoelasticity of zeroth order. The analytical treatment, however, is manageable only for the case of principal wave fronts for which the spatial direction of propagation coincides with a principal direction of the initial state of stress and, consequently, the wave fronts separate into longitudinal and transverse ones. Such notions as those of apparent elasticity moduli appear naturally in the analysis. Then a model of thermodynamical relativistic elasticity, referred to as neo-Hookean elasticity, is shown to be representable, insofar as wave-front propagation is concerned, by a special model of hypoelasticity of first order. The qualitative results obtained before concerning the influence of initial stresses are shown to apply equally to this description.
\end{abstract}

\section{Introduction}

The interest of relativistic elasticity for the study of the deformation of massive stellar objects on the one hand [1-4], and for a coherent approach to the vibrations of elastic detectors of gravitational waves on the other hand $[5,6]$, has been emphasized in recent years. The recent history of relativistic elasticity offers two avenues of development, one initiated by Synge [7], and the other that makes use of thermodynamical arguments and is illustrated, for instance, by the already quoted papers. In formulating his pioneering theory of relativistic elasticity, Synge sought to avoid the definition of an initially stress-free state which, because of the ever operating gravitational field, cannot exist. However, recent developments in relativistic continuum mechanics have placed in evidence some shortcomings of his formulation (no relation to thermodynamics, noninvariance of the constitutive equations with respect to the observers, i.e., "nonobjectivity"). Furthermore, it 
appears that in Synge's work and in those of the authors who took over his formulation (cf. $[8,9]$ ) no influence whatsoever of an initial state of stress shows up in the properties of wave-front propagation (e.g., propagation speeds independent of initial stresses). Therefore, the purpose of the present study is threefold: First, to recall what should be a "correct" formulation of relativistic elasticity in the tradition of Synge, thus a formulation of so-called relativistic hypoelasticity (cf. [10]); next, to reconciliate such a formulation with thermodynamical arguments; finally, to exhibit the alterations brought by a general initial state of stress in the propagation process of so-called infinitesimal discontinuities (alteration in the wave speeds, existence of three different speeds in general and the separation of wave fronts in one longitudinal and two transverse wave fronts with different propagation speeds in the case where the direction of propagation coincides with a proper direction of the initial state of stress, the growth of infinitesimal discontinuities so as to form a shock). The study of infinitesimal discontinuities makes a systematic use (i) of the elegant formalism due to Lichnerowicz [11] and (ii) of canonical space-time decompositions and of spatial decompositions along the propagation direction of the wave front, and onto the two-dimensional hyperplane orthogonal to it, which allows us to keep the covariant formalism as long as possible. The present relativistic study has many points in common with the now classical study of infinitesimal perturbations superimposed on a finite state of stress in classical continuum mechanics (see, e.g., [12]).

The notation used, the statement of the general field equations and of general classes of constitutive equations, and the definition of weak discontinuity fronts in the relativistic framework are given in Section 2. Section 3 is devoted to the study of the simplest case, namely, that of the propagation of infinitesimal discontinuities in relativistic hypoelastic bodies of zeroth order. The wave-front speeds are determined exactly in the case of so-called principal wave fronts, for which the spatial direction of propagation coincides with a proper direction of the initial state of stress. There follows a short discussion for the general case where this simplifying assumption does not hold good. Then the distorsion of signals for principal longitudinal wave fronts is exhibited by using Lichnerowicz's qualitative method [11]. In Sections 4 and 5 it is shown that relativistic neo-Hookean elasticity can be reduced to the scheme of relativistic hypoelasticity of the first order insofar as wave propagation superimposed on an initial state of stress is concerned. Indeed, both a direct treatment in terms of strains and a treatment using an appropriate stressstrain relation of the hypoelastic type as intermediary yield the same conclusions as to the wave-front speeds. By way of conclusion, in Section 6, we remark upon the limitations inherent in the present approach which, therefore, should be supplemented with an exact thermodynamical treatment.

\section{Notation, General Equations}

\subsection{Space-Time, Differentiation}

Let $M=\left(V^{4}, g_{\alpha \beta}\right)$ be a space-time of general relativity equipped with a normal hyperbolic metric $g_{\alpha \beta}(\alpha, \beta=1,2,3,4$; index 4 time-like; Lorentzian signature,++ , $+,-)$. The field of world velocity $u^{\alpha}$, such that $g_{\alpha \beta} u^{\alpha} u^{\beta}=-1(c=1$ for notational 
convenience) defines the invariant derivative $D \equiv u^{\alpha} \nabla_{\alpha}$. It also defines the field of spatial projectors $P_{\alpha \beta}=g_{\alpha \beta}+u_{\alpha} u_{\beta}$, such that $P_{\alpha \beta} u^{\beta}=0$ and $P_{\alpha}^{\alpha}=3$. The latter in turn serves to write down the local canonical space-time decomposition of any tensor field defined on $M$. In particular, the spatial projection of such a tensor field obtained by applying the spatial projector is noted $(\ldots)_{\perp}$. Geometrical objects $\boldsymbol{A}$ which satisfy $\boldsymbol{A} \equiv(\boldsymbol{A})_{\perp}$ are said to be spatial (or PU: orthogonal to $\boldsymbol{u}$ [13]). In particular, the transverse or spatial covariant derivative is defined by

$$
\stackrel{1}{\nabla}_{\alpha} \equiv P_{\alpha}^{\beta} \nabla_{\beta}=\nabla_{\alpha}+u_{\alpha} D, \quad\left(u^{\alpha} \vec{\nabla}_{\alpha}\right) \rightarrow 3
$$

The spatial rate of strain is defined by

$$
d_{\alpha \beta}=\frac{1}{2}\left(\frac{1}{\nabla}_{\alpha} u_{\beta}+\stackrel{1}{\nabla}_{\beta} u_{\alpha}\right) \equiv \stackrel{1}{\nabla}_{(\alpha} u_{\beta)} ;
$$

and a simple computation allows us to check that

$$
\mathfrak{u}_{u} P_{\alpha \beta}=2 d_{\alpha \beta}
$$

on account of Ricci's lemma and of the definition of the Lie derivative along the field $u^{\alpha}$, noted $\underset{u}{£}$, for a covariant spatial tensor field. E.g., if $A_{\alpha \beta}$ is spatial,

$$
(\underset{u}{\mathfrak{A} A})_{\perp \alpha \beta}=\left(D A_{\alpha \beta}\right)_{\perp}+A_{\nu \beta} \frac{1}{\nabla_{\alpha}} u^{\gamma}+A_{\alpha \gamma} \frac{1}{\nabla_{\beta}} u^{\gamma} .
$$

Setting $d_{\alpha \beta}=0$ defines a so-called Herglotz-Born rigid-body motion (in differential form; Killing's theorem).

\subsection{Field Equations}

In supplement to Einstein's field equations that relate linearly the Einstein tensor and the total energy-momentum tensor, we have

(a) the equation of continuity:

$$
\nabla_{\alpha}\left(\varrho u^{\alpha}\right)=0
$$

or

$$
D \varrho+\varrho d_{\cdot \gamma}^{\gamma}=0
$$

(b) the "conservation" of energy-momentum (consequence of Bianchi's identity):

$\nabla_{\beta} T^{\alpha \beta}=0 ;$

(c) the "conservation" of moment of energy-momentum in absence of spin:

$$
T^{[\alpha \beta]} \equiv \frac{1}{2}\left(T^{\alpha \beta}-T^{\beta \alpha}\right)=0 \text {. }
$$

The scalar field $\varrho$ represents the mass per unit of proper volume. Then, in absence of heat conduction, electromagnetic fields and spin, $T^{\alpha \beta}$ admits the following simplified canonical space-time decomposition:

$$
T^{\alpha \beta}=\varrho \omega u^{\alpha} u^{\beta}-t^{\alpha \beta},
$$


where the spatial tensor $t^{\alpha \beta}$ is the relativistic stress tensor and $\omega$ has the expression

$$
\omega=1+\varepsilon,
$$

where the first contribution represents the rest energy and $\varepsilon$ is the internal energy per unit of proper mass.

Taking account of (2.5), using the fact that $t^{\alpha \beta}$ is symmetrical as a consequence of (2.8), we contract (2.7) with $u_{\alpha}$ to obtain the energy equation

$$
\varrho D \varepsilon-t^{\alpha \beta} d_{\alpha \beta}=0 \text {. }
$$

Applying the spatial projector to (2.8), noting that $D u^{\alpha} \equiv\left(D u^{\alpha}\right)_{\perp}$, and using the definition (2.1), we obtain the Euler-Cauchy equations of motion in the form

$$
\varrho f_{. \beta}^{\alpha} D u^{\beta}-P_{. \gamma}^{\alpha} \bar{D}_{\beta} t^{\gamma \beta}=0 \text {. }
$$

These represent only three independent scalar equations in virtue of the spatial character. The spatial symmetrical tensor of mixed components

$$
f_{. \beta}^{\alpha} \equiv \omega P_{. \beta}^{\alpha}-Q^{-1} t_{. \beta}^{\alpha}
$$

will be referred to as the tensorial index of the material continuum ${ }^{1}$.

\subsection{Constitutive Equations}

We shall consider the following general class of constitutive equations for relativistic (so-called) hypoelastic solids: The relativistic stress tensor $t_{\alpha \beta}$ and the rate of strain tensor $d_{\alpha \beta}$ are related by an equation of the general type ([10], cf. pp. 140 145):

$$
(\mathscr{D} \boldsymbol{t})_{\perp}=\mathscr{L}[\boldsymbol{d} ; \boldsymbol{t}] .
$$

Here $\mathscr{D}$ is a time-like differential operator and $\mathscr{L}$ is a spatial symmetric covariant operator which acts linearly on $\boldsymbol{d}$, which is continuously differentiable in the neighborhood of $\boldsymbol{d}=0$, and is jointly isotropic in $\boldsymbol{d}$ and $\boldsymbol{t}^{2}$. The condition of isotropy and the fact that $\mathscr{D}$ must define a so-called objective time-like derivative follow necessarily if it is posited that constitutive equations of the rate-type form (2.14) be form-invariant under change of observer, or, in other words, be either objective in agreement with the axiom set forth in Ref. [15], or rheologically invariant according to the terminology of Oldroyd [16].

\footnotetext{
1 This generalizes the notion of index, a thermodynamical function introduced by Lichnerowicz [14], to general continua (i.e., continua whose stress tensor is not necessarily spherical). For perfect fluids we have $t_{. \beta}^{\alpha}=-p P_{. \beta}^{\alpha}$, where $p$ is the thermodynamical pressure, and the definition (2.13) reduces to $f_{. \beta}^{\alpha}=f P_{. \beta}^{\alpha}$ where

$$
f=1+i=1+(\varepsilon+p / \varrho)
$$

is the scalar index used by Lichnerowicz in relativistic hydrodynamics

2 This notion of isotropy is not to be mistaken for that of isotropy as applied to four-vectors of zero magnitude in space-time. Here isotropy is understood in the classical sense, meaning isotropy in $\mathbb{E}^{3}$, i.e., invariance under $\mathrm{SO}(3)$. The necessary and sufficient condition for isotropy is established by writing (2.14) in nonholonomic components on a spatial triad (completed by $\boldsymbol{u}$ to form a space-time tetrad)--a special case is the use of Fermi coordinates. This invariance can be formulated in the language of spinors
} 
Several remarks are in order concerning constitutive equations of the type (2.14). Firstly, equations such as (2.14) have an incremental character (suited for computations on computers) and need initial conditions, in particular, the knowledge of initial stresses, to be integrated. Without such initial conditions, Equation (2.14) define only a class of continua. Secondly, having the nature of ratetype equations, they do not involve strains per se. The definition of such strains in a general relativistic framework is thus avoided. These are two arguments that favored the introduction of an equation of a simple type related to (2.14) by Synge in 1959 [7], although Synge's proposal appears too simple in the light of recent developments in the relativistic mechanics of continua. Next, thermodynamical considerations apparently do not enter the establishment of equations of this kind. However, in special circumstances, i.e., for a very special expression of $\varepsilon$, it can be shown that Equations (2.14) are none other than the differentiated form of thermodynamical constitutive equations which relate the relativistic stress tensor and a finite-strain tensor (see below, Section 4). In spite of the shortcoming just mentioned concerning the (general) lack of thermodynamical justification for equations of the type (2.14), we note that, from a pragmatic viewpoint, such equations will yield, conveniently and at once, compatibility conditions between the infinitesimal discontinuities in the stresses and the four-velocity. Such conditions will involve the initial stresses if $t$ is continuous (see herebelow). A hypoelastic body with constitutive equations (2.14) is necessarily not viscous ${ }^{3}$. Finally, the definition (2.14) must be given a more precise form. For instance, on account of the properties of objectivity, linearity and isotropy, a possible expression, of which the right-hand side does not depend on $\boldsymbol{t}$, is

$$
{ }^{*} t_{\alpha \beta}=\mathscr{L}_{\alpha \beta}^{. \mu \nu} d_{\mu \nu},
$$

where a superimposed asterisk denotes the convective time derivative defined by

$$
{ }^{*} t_{\alpha \beta} \equiv\left(\mathfrak{f}_{u} t_{\alpha \beta}\right)_{\perp}+t_{\alpha \beta} d_{. \mu}^{\mu}
$$

and the components $\mathscr{L}_{\alpha \beta}^{. \mu \nu}$ of the linear operator $\mathscr{L}$ have the expression

$$
\mathscr{L}_{\alpha \beta}^{. \mu \nu}=\lambda P_{\alpha \beta} P^{\mu v}+2 \mu P_{\alpha}^{. \mu} P_{\beta}^{. v},
$$

where $\lambda$ and $\mu$ are two scalars characteristic of the material, which are linear in $\varrho$. That is, we can introduce $\tilde{\lambda}$ and $\tilde{\mu}$ such that

$$
\lambda=\varrho \tilde{\lambda}, \quad \mu=\varrho \tilde{\mu} \quad(\underset{u}{\mathfrak{X}} \tilde{\lambda}=\underset{u}{\mathfrak{\mu}} \tilde{\mu}=0) .
$$

Hypoelastic continua with constitutive equations (2.15)-(2.18) are called $h y$ poelastic bodies of zeroth order. If, however, the right-hand side of (2.14) is affine in $\boldsymbol{t}$, then it can be shown that the expression of $\mathscr{L}_{\alpha \beta}^{. . \mu v}$ reads

$$
\begin{aligned}
& \mathscr{L}_{\alpha \beta}^{. \mu \nu}=\varrho\left[\left(\tilde{\lambda}+\tilde{\lambda}^{\prime} t_{. \gamma}^{\gamma}\right) P_{\alpha \beta} P^{\mu \nu}+2\left(\tilde{\mu}+\tilde{\mu}^{\prime} t_{. \gamma}^{\gamma}\right) P_{\alpha}^{. \mu} P_{\beta}^{. v}+\tilde{\alpha} P_{\alpha \beta} t^{\mu \nu}\right. \\
& \left.+\tilde{\beta} t_{\alpha \beta} P^{\mu v}+\tilde{\gamma}\left(P_{\alpha}^{. \mu} t_{\beta}^{v}+P_{\alpha}^{. v} t_{\beta}^{\mu}+P_{\beta}^{. \mu} t_{\alpha}^{\cdot v}+P_{\beta}^{\cdot{ }^{v}} t_{\alpha}^{\mu}\right)\right],
\end{aligned}
$$

3 This follows from the invariance of (2.14) under scaling of the proper time, llence there cannot be relaxation times in $(2.14)$ 
where, $\tilde{\lambda}, \tilde{\lambda}^{\prime}, \tilde{\mu}, \tilde{\mu}^{\prime}, \tilde{\alpha}, \tilde{\beta}$, and $\tilde{\gamma}$ are seven scalars characteristic of the material. Hypoelastic continua with constitutive equations (2.15), (2.16), and (2.19) are called hypoelastic bodies of first order ${ }^{4}$.

\subsection{Discontinuity Fronts $[11,19]$}

Let $W\left(x^{\alpha}\right)=0$ be the equation of a discontinuity front-a time-like hypersurfacewhich propagates in $V^{4}$ and which separates the region $\mathscr{B}$ of $V^{4}$ swept out by the matter in motion in two subregions $\mathscr{B}^{+}$and $\mathscr{B}^{-}$at each time. We set

$$
l_{\alpha}=\partial_{\alpha} W, \quad L=\left(P^{\alpha \beta} l_{\alpha} l_{\beta}\right)^{1 / 2},
$$

and

$$
\lambda_{\alpha} \equiv L^{-1} P_{\alpha}^{. \beta} l_{\beta}, \quad\left(P^{\alpha \beta} \lambda_{\alpha} \lambda_{\beta}=1\right),
$$

so that

$$
l_{\alpha}=L\left(\lambda_{\alpha}-\mathscr{U} u_{\alpha}\right)
$$

if

$$
\mathscr{U} \equiv L^{-1}\left(u^{\sigma} l_{\sigma}\right) .
$$

$\lambda_{\alpha}$ is a unit spatial covariant vector field. $\mathscr{U}$ is the (nondimensional) speed of the discontinuity front measured relatively to the moving matter. $l_{\alpha}$ being oriented from the "minus" side to the "plus" side of $W$, we note $\llbracket \boldsymbol{A} \rrbracket=\boldsymbol{A}^{+}-\boldsymbol{A}^{-}$, where $\boldsymbol{A}^{+}$and $\boldsymbol{A}^{-}$ are the uniform limits of $\boldsymbol{A}$ in approaching $W$ on its two faces. Let $\boldsymbol{A}$ be continuous across $W \bar{\delta}$ being the Dirac distribution with support $W$, the infinitesimal discontinuity of $\boldsymbol{A}$, noted $\delta \boldsymbol{A}$, is given by [11]

$$
\bar{\delta} \llbracket \nabla_{\alpha} A \rrbracket=l_{\alpha} \delta \boldsymbol{A} .
$$

Then

$$
\bar{\delta} \llbracket \stackrel{\perp}{\nabla}_{\alpha} A \rrbracket=L \lambda_{\alpha} \delta \boldsymbol{A}, \quad \bar{\delta} \llbracket D A \rrbracket=L \mathscr{U} \delta \boldsymbol{A},
$$

if $u^{\alpha}$ is continuous across $W$.

The canonical decomposition, along $\lambda_{\alpha}$ and onto the two-dimensional space tangent to the spatial part of $W$ and orthogonal to $\lambda$, of any spatial tensor field is obtained by using the two-dimensional (spatial) projector

$$
S_{\alpha \beta} \equiv P_{\alpha \beta}-\lambda_{\alpha} \lambda_{\beta}=S_{\beta \alpha}, \quad S_{\alpha \beta} \lambda^{\beta}=S_{\alpha \beta} u^{\beta}=0, \quad S_{. \alpha}^{\alpha}=2 .
$$

For instance, let $\delta u^{\alpha}$ and $\delta t^{\alpha \beta}$ be the infinitesimal discontinuities in the four-velocity and the relativistic stress tensor. Then we have the decompositions

$$
\delta u^{\alpha}=\delta u_{\perp}^{\alpha}+\lambda^{\alpha} \delta u
$$

and

$$
\left(\delta t^{\alpha \beta}\right)_{\perp}=\delta \tau^{\alpha \beta}+\delta \tau^{\alpha} \lambda^{\beta}+\delta \tau^{\beta} \lambda^{\alpha}+\delta \tau \lambda^{\alpha} \lambda^{\beta},
$$

4 Comments on the physical validity of models of classical continua having constitutive equations which are the nonrelativistic versions of Equation (2.15) are to be found in Eringen [17], p. 331, and Truesdell and Noll [18], p. 405 
where

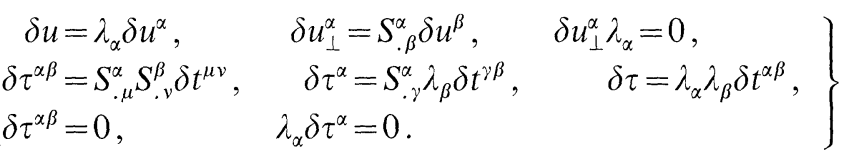

Remark that $\delta u^{\alpha}$ is purely spatial and $\delta u_{\perp}^{\alpha}$ is essentially a two-dimensional geometrical object, but expressed in a fully covariant form on $M$. Similarly to Equation (2.28), if $t^{\alpha \beta}$ is a second order symmetric spatial tensor field which is continuous across $W$, we have the following canonical decomposition at any event point $\boldsymbol{x} \in W$ :

$$
t^{\alpha \beta}=\bar{T}^{\alpha \beta}+2 \bar{T}^{(\alpha} \lambda^{\beta)}+\bar{T} \lambda^{\alpha} \lambda^{\beta} .
$$

If $\varepsilon, \varrho$, and $t^{\alpha \beta}$ are continuous across $W$, then an analogous decomposition holds true for the tensorial index:

$$
f_{\alpha \beta}=\bar{F}_{\alpha \beta}+2 \bar{F}_{(\alpha} \lambda_{\beta)}+\bar{F} \lambda_{\alpha} \lambda_{\beta} .
$$

It is a simple matter to check that

$$
\bar{F}_{\alpha \beta}=\omega S_{\alpha \beta}-\varrho^{-1} \bar{T}_{\alpha \beta}, \quad \bar{F}_{\alpha}=-\varrho^{-1} \bar{T}_{\alpha}, \quad \bar{F}=\omega-\varrho^{-1} \bar{T},
$$

the metric used to raise and lower indices being assumed continuous across $W$. In fact, we shall consider the following hypotheses:

$H_{1}: u^{\alpha}, \varrho, \varepsilon, t^{\alpha \beta}$ are continuous across $W$;

$H_{2}$ : space-time derivatives of these fields suffer discontinuities across $W$;

$\mathrm{H}_{3}$ : the wave front $W$ is not a gravitational wave front. The wave equation of such a wave front would be $g^{\alpha \beta} l_{\alpha} l_{\beta}=0$ or, using (2.22), $\mathscr{U}^{2}=1$;

$H_{4}$ : the wave front $W$ is not material i.e., is not generated by the trajectories of the matter. Hence $\mathscr{U} \neq 0$ from here on ${ }^{5}$.

The last two conditions and relativistic causality limit the range of $\mathscr{U}$ to the open interval $] 0,1[$.

Wave fronts $W$ for which $\left(\delta u \neq 0, \delta u_{\perp}^{\alpha}=0\right)$ are called longitudinal wave fronts whereas wave fronts for which $\left(\delta u=0, \delta u_{\perp}^{\alpha} \neq 0\right)$ will be referred to as transverse wave fronts. Wave fronts for which $\delta u$ and $\delta u_{\perp}^{\alpha}$ differ from zero simultaneously are called general or mixed wave fronts.

\section{Infinitesimal Discontinuities in Relativistic Hypoelastic Bodies of Zeroth Order}

\subsection{Equations Governing the Discontinuities}

Consider the system of field equations formed by Equations (2.6), (2.11), (2.12), and (2.15)-(2.18). That is,

$$
\begin{aligned}
& D \varrho+\varrho d_{\cdot \gamma}^{\gamma}=0, \\
& \varrho D \varepsilon-t^{\alpha \beta} d_{\alpha \beta}=0, \\
& \varrho f_{. \beta}^{\alpha} D u^{\beta}-P_{\cdot \gamma}^{\alpha} \frac{1}{\nabla_{\beta}} t^{\gamma \beta}=0,
\end{aligned}
$$

5 Using a thermodynamical background shows that $\mathscr{U}=0$ corresponds to entropy wave fronts (cf. Section 4) 
and

$$
\left(D t_{\alpha \beta}\right)_{\perp}+t_{\gamma \beta} \frac{1}{\nabla_{\alpha}} u^{\gamma}+t_{\alpha \gamma} \stackrel{1}{\nabla}_{\beta} u^{\gamma}+t_{\alpha \beta} \nabla_{\gamma} u^{\gamma}=\lambda d_{. \gamma}^{\gamma} P_{\alpha \beta}+2 \mu d_{\alpha \beta} .
$$

Under the hypotheses set forth in the foregoing paragraph, with $\mathscr{U} \neq 0$, we deduce from these equations the following discontinuity-governing equations:

$$
\begin{aligned}
& \delta \varrho=-\varrho \mathscr{U}^{-1} \delta u, \\
& \delta \varepsilon=\frac{1}{2}(\varrho \mathscr{U})^{-1} t^{\alpha \beta}\left(\lambda_{\alpha} \delta u_{\beta}+\lambda_{\beta} \delta u_{\alpha}\right), \\
& \varrho \mathscr{U} f^{\alpha \beta} \delta u^{\beta}-\lambda_{\beta}\left(\delta t^{\alpha \beta}\right)_{\perp}=0, \\
& \mathscr{U}\left(\delta t_{\alpha \beta}\right)_{\perp}=\lambda P_{\alpha \beta} \delta u+\mu\left(\lambda_{\alpha} \delta u_{\beta}+\lambda_{\beta} \delta u_{\alpha}\right)-\lambda_{\alpha} t_{\beta \mu} \delta u^{\mu} \\
& \quad-t_{\alpha \mu} \lambda_{\beta} \delta u^{\mu}-t_{\alpha \beta} \delta u .
\end{aligned}
$$

Equation (3.8) is the compatibility condition which relates $\left(\delta t_{\alpha \beta}\right)_{\perp}$ and $\delta u^{\alpha}$.

Applying the decomposition procedure along $\lambda$ and orthogonally to $\lambda$ to Equations (3.7) on account of (2.27), (2.28), (2.30), and (2.32), we obtain

$$
\delta \tau=\varrho \mathscr{U}\left(\bar{F}_{\alpha} \delta u_{\perp}^{\alpha}+\bar{F} \delta u\right),
$$

and

$$
\delta \tau^{\alpha}=\varrho \mathscr{U}\left(\bar{F}_{. \beta}^{\alpha} \delta u_{\perp}^{\beta}+\bar{F}^{\alpha} \delta u\right) .
$$

Similarly, Equation (3.8) yields

$$
\begin{aligned}
& \mathscr{U} \delta \tau+2 \bar{T}_{\alpha} \delta u_{\perp}^{\alpha}-(\lambda+2 \mu-3 \bar{T}) \delta u=0, \\
& \mathscr{U} \delta \tau^{\alpha}+\left(\bar{T}_{. \beta}^{\alpha}-\mu S_{. \beta}^{\alpha}\right) \delta u_{\perp}^{\beta}+2 \bar{T}^{\alpha} \delta u=0,
\end{aligned}
$$

and

$$
\mathscr{U} \delta \tau^{\alpha \beta}+\left(\bar{T}^{\alpha \beta}-\lambda S^{\alpha \beta}\right) \delta u=0 .
$$

The latter can be rewritten as

$$
\delta \tau^{\alpha \beta}=\mathscr{U}^{-1}\left(\lambda S^{\alpha \beta}-\bar{T}^{\alpha \beta}\right) \delta u .
$$

By the same token Equation (3.6) takes on the form

$$
\delta \varepsilon=(\varrho \mathscr{U})^{-1}\left(\bar{T}_{\alpha} \delta u^{\alpha}+\bar{T} \delta u\right) .
$$

Let $g_{\alpha \beta}^{(0)}$ and

$$
\mathfrak{M}_{0}(\boldsymbol{x} \in \mathscr{B} \subset M)=\left\{\varrho, \varepsilon, u^{\alpha}, t^{\alpha \beta}\right\}
$$

be a solution of the system of field equations. Then Equations (3.5), (3.15), (3.9), (3.10) and (3.14) provide, at each event point $\boldsymbol{x} \in W$, the values of the infinitesimal discontinuities $\delta \varrho, \delta \varepsilon, \delta \tau, \delta \tau^{\alpha}$, and $\delta \tau^{\alpha \beta}$ as functions of $\mathfrak{M}_{0}$ and of the wave speed $\mathscr{U}$, and as linear expressions in the strength of the wave front. This strength is represented by both the scalar $\delta u$ and the essentially two-dimensional vector field $\delta u_{\perp}^{\alpha}$, and is given by initial conditions concerning the wave front. We can write 
formally

$$
\begin{aligned}
& \delta \varrho=f_{(\varrho)}\left[(\delta u, 0) ; \mathscr{U}, \mathfrak{M}_{0}\right], \\
& \left(\delta \varepsilon, \delta \tau, \delta \tau^{\alpha}\right)=f_{\left(\varepsilon, \tau, \tau^{\alpha}\right)}\left[\left(\delta u, \delta u_{\perp}^{\alpha}\right) ; \mathscr{U}, \mathfrak{M}_{0}\right], \\
& \delta \tau^{\alpha \beta}=f_{\left(\tau^{\alpha \beta}\right)}\left[(\delta u, 0) ; \mathscr{U}, \mathfrak{M}_{0}\right] .
\end{aligned}
$$

Obviously, the infinitesimal discontinuities $\delta \varrho$ and $\delta \tau^{\alpha \beta}$ are zero only for pure transverse waves while nonzero $\delta \varepsilon, \delta \tau$, and $\delta \tau^{\alpha}$ exist for all types of wave front under general conditions of initial stresses.

On account of the remark just made it remains to exploit Equations (3.11) and (3.12) on account of Equations (3.9) and (3.10). Eliminating $\delta \tau$ and $\delta \tau^{\alpha}$ between these equations, we arrive at the following system of coupled equations (linear in the wave-front strength)

$$
\begin{aligned}
& \left(\varrho \mathscr{U}^{2} \bar{F}_{. \beta}^{\alpha}+\bar{T}_{. \beta}^{\alpha}-\mu S_{. \beta}^{\alpha}\right) \delta u_{\perp}^{\beta}+\left(\varrho \mathscr{U}^{2} \bar{F}^{\alpha}+2 \bar{T}^{\alpha}\right) \delta u=0, \\
& \left(\varrho \mathscr{U}^{2} \bar{F}_{\beta}+2 \bar{T}_{\beta}\right) \delta u_{\perp}^{\beta}+\left[\varrho \mathscr{U}^{2} \bar{F}-(\lambda+2 \mu-3 \bar{T})\right] \delta u=0 .
\end{aligned}
$$

We postpone the study of this general system until $\S 3.3$, to study first a special case of propagation for which the polarization vector $\lambda_{\alpha}$ is along a preferred spatial direction.

\subsection{Principal Wave Fronts}

In general $t_{\alpha \beta}$ admits three distinct eigenvectors $d_{(k)}^{\beta}, k=1,2,3$, with corresponding eigenvalues $t_{(k)}$ in such a way that (no summation on $k$ )

$$
t_{\alpha \beta} d_{(k)}^{\beta}=t_{(k)} d_{(k) \alpha}, \quad d_{(k) \alpha} \equiv P_{\alpha \beta} d_{(k)}^{\beta},
$$

and

$$
P^{\alpha \beta} d_{(k) \alpha} d_{(k) \beta}=1, \quad P^{\alpha \beta} d_{(k) \alpha} d_{(l) \beta}=\delta_{k l} .
$$

We call principal wave fronts those wave fronts for which $\lambda$ coincides with one of the eigenvectors $\boldsymbol{d}_{(k)}$, for instance, $\boldsymbol{d}_{(1)}$. Let $t_{(1)}=t_{\|}$be the corresponding stress eigenvalue. It then follows from (2.30)-(2.32) that

$$
\bar{T}^{\alpha}=\bar{F}^{\alpha} \equiv 0,
$$

and

$$
\bar{T}=t_{\|}, \quad \bar{F}=\omega-\varrho^{-1} t_{\|} .
$$

In this case Equations (3.20) and (3.21) uncouple and read

$$
\left(\varrho \mathscr{U}^{2} \bar{F}_{. \beta}^{\alpha}+\bar{T}_{. \beta}^{\alpha}-\mu S_{. \beta}^{\alpha}\right) \delta u_{\perp}^{\beta}=0,
$$

and

$$
\left[\varrho \mathscr{U}^{2} \bar{F}-(\lambda+2 \mu-3 \bar{T})\right] \delta u=0 .
$$


If $\delta u$ is not zero, i.e., for longitudinal principal wave fronts, (3.27) implies that the wave speed $\mathscr{U}=\mathscr{U}_{L 1}$ is given by

$$
\mathscr{U}_{L 1}^{2}=\left(\lambda+2 \mu-3 t_{||}\right) / \varrho\left(1+\varepsilon-\varrho^{-1} t_{||}\right) .
$$

Relativistic causality implies, with the choice $\mathscr{U}_{L 1}>0$, that $\lambda, \mu, \varrho, \varepsilon$, and $t_{\|}$satisfy the constraint $\mathscr{U}_{L 1}<1$. Of course, $\mathscr{U}_{L 1}$ depends on $\mathfrak{M}_{0}$. Then, with $\delta u_{\perp}^{\alpha}=0$, Equations (3.17) through (3.19) provide the infinitesimal discontinuities $\left(\delta \varrho, \delta \varepsilon, \delta \tau, \delta \tau^{\alpha}, \delta \tau^{\alpha \beta}\right)$ in the form

$$
\begin{aligned}
& \delta \varrho=-\varrho \mathscr{U}_{L 1}^{-1} \delta u, \quad \delta \varepsilon=\left(\varrho \mathscr{U}_{L 1}\right)^{-1} t_{\|} \delta u, \\
& \delta \tau=\varrho \mathscr{U}_{L 1}\left(1+\varepsilon-\varrho^{-1} t_{\|}\right) \delta u, \quad \delta \tau^{\alpha}=0,
\end{aligned}
$$

and

$$
\delta \tau^{\alpha \beta}=\mathscr{U}_{L 1}^{-1}\left(\lambda S^{\alpha \beta}-\bar{T}^{\alpha \beta}\right) \delta u .
$$

In the nonrelativistic limit and for an initially unstressed body, Equation (3.28) reduces to the well-known Lamé value $\mathscr{U}_{L}^{2}=(\lambda+2 \mu) / \varrho$.

If $\delta u_{\perp}^{\alpha} \neq 0$, then Equation (3.26) implies that the $2 \times 2$ determinant of the factor of $\delta u_{\perp}^{\beta}$ be zero, i.e.,

$$
\underset{2 \times 2}{\operatorname{det}}\left|\varrho \mathscr{U}^{2} \bar{F}_{. \beta}^{\alpha}+\bar{T}_{. \beta}^{\alpha}-\mu S_{. \beta}^{\alpha}\right|=0 .
$$

This can be solved easily if we consider a local chart in which coordinate axes of the two-dimensional hyperplane $H_{(\lambda)}$ orthogonal to $\lambda$ are directed along the remaining two eigenvectors of $t_{\alpha \beta}$, i.e., along $\boldsymbol{d}_{(2)}$ and $\boldsymbol{d}_{(3)}$ with corresponding eigenvalues $t_{(2)}$ and $t_{(3)}$ for $t_{\alpha \beta}$, and $S_{\alpha \beta}=\operatorname{diag}(+1,+1)$. Then Equation (3.32) yields two different speeds for transverse wave fronts as, on account of relativistic causality,

$$
\mathscr{U}_{T 2}^{2}=\frac{\mu-t_{(2)}}{\varrho(1+\varepsilon)-t_{(2)}}<1, \quad \mathscr{U}_{T 3}^{2}=\frac{\mu-t_{(3)}}{\varrho(1+\varepsilon)-t_{(3)}}<1 .
$$

For $\delta u=0$ and on account of (3.24) the only nonzero infinitesimal discontinuity is that given by (3.10), i.e.,

$$
\delta \tau_{(2,3)}^{\alpha}=\varrho \mathscr{U}_{T 2,3} \bar{F}_{. \beta}^{\alpha} \delta u_{\perp}^{\beta} .
$$

In the nonrelativistic limit and for an initially unstressed body, both wave speeds (3.33) coalesce to the well-known Lamé value $\mathscr{U}_{T}^{2}=\mu / \varrho$.

Other special cases can be considered. For instance, the case where $t_{\alpha \beta}$ corresponds to a uniaxial state of stress is a degenerate case for which $t_{(2)}$ and $t_{(3)}$ are equal to the same value $t_{\perp}$. Then $t_{\alpha \beta}$ has the expression

$$
t_{\alpha \beta}=t_{\perp} S_{\alpha \beta}+t_{||} \lambda_{\alpha} \lambda_{\beta}=t_{\perp} P_{\alpha \beta}+\left(t_{\|}-t_{\perp}\right) \lambda_{\alpha} \lambda_{\beta} .
$$

Then the remaining two eigendirections are arbitrary in $H_{(\lambda)}$ insofar as they remain orthogonal to each other (this results from the invariance of $t_{\alpha \beta}$ by rotation about $\lambda$ ). Equation (3.33) then reduce to the single equation

$$
\mathscr{U}_{T}^{2}=\left(\mu-t_{\perp}\right) /\left[\varrho(1+\varepsilon)-t_{\perp}\right] .
$$


The completely degenerate case is that for which $t_{\alpha \beta}$ is spherical and corresponds to a state of hydrostatic pressure:

$$
t_{\alpha \beta}=-p_{0} P_{\alpha \beta}=-p_{0} S_{\alpha \beta}-p_{0} \lambda_{\alpha} \lambda_{\beta} .
$$

The last equation shows that it is always possible to consider this case as a special case of principal-wave-front propagation. Then $\bar{T}=-p_{0}, \bar{T}^{\alpha \beta}=-p_{0} S^{\alpha \beta}$, and $\bar{F}_{\alpha \beta}$ $=f_{0} S_{\alpha \beta}$ with $f_{0}=1+\varepsilon+\left(p_{0} / \varrho\right)$. Remark that $f_{0}=1+0\left(c^{-2}\right)$. Thus,

$$
\mathscr{U}_{L}^{2}=\bar{\mu} / \varrho f_{0}, \quad \mathscr{U}_{T 2}^{2}=\mathscr{U}_{T 3}^{2}=(\bar{\lambda}+2 \bar{\mu}) / \varrho f_{0},
$$

where

$$
\bar{\mu} \equiv \mu+p_{0}=\varrho\left(\tilde{\mu}+p_{0} / \varrho\right), \quad \bar{\lambda} \equiv \lambda+p_{0}=\varrho\left(\tilde{\lambda}+p_{0} / \varrho\right)
$$

are apparent elasticities ${ }^{6}$. This notion of apparent elasticity modulus in an initially pressurized body is common in classical seismology (cf. [20], p. 253).

The results just obtained bring about the following comments. In hypoelastic bodies described by the constitutive Equations (2.15)-(2.18), a wave front travelling down a principal axis of stress is always either longitudinal or transverse. For a general initial state of stress, the two wave speeds corresponding to transverse wave fronts are different. Transverse wave fronts with amplitude parallel to the axis of lesser transverse stress travel at greater absolute speed than the others. These two kinds of transverse waves travel at the same absolute speed if and only if the corresponding eigenstresses are equal. In this case, any transverse amplitude is admissible. These comments parallel those made by Truesdell [22] in his study of wave fronts in classical nonlinear isotropic elasticity. Finally, relativistic causality imposes constraints not only on the moduli $\lambda$ and $\mu$, but rather on combinations of $\lambda, \mu$ and of initial values of energy, density and stresses. This shows that in the case of initially stressed relativistic elastic bodies, the hyperbolicity is much more difficult to study than recent studies tend to demonstrate (cf. $[8,9])$. Such a study is required, but will not be attacked here.

Two final remarks concern the general result (3.33)and prove one of the abovemade comments. Firstly, forming $\mathscr{U}_{T 2}^{2}-\mathscr{U}_{T 3}^{2}$, we get

$$
\mathscr{U}_{T 2}^{2}-\mathscr{U}_{T 3}^{2}=\frac{[\varrho(1+\varepsilon)-\mu]\left[t_{(3)}-t_{(2)}\right]}{\left[\varrho(1+\varepsilon)-t_{(2)}\right]\left[\varrho(1+\varepsilon)-t_{(3)}\right]} .
$$

Remark that each term in the denominator of this expression is greater than zero since each of these terms has the form $\varrho\left[1+0\left(c^{-2}\right)\right]$. Hence, if, in our units, $\varrho(1+\varepsilon)$ $>\mu$, then $t_{(3)}>t_{(2)}$ implies $\mathscr{U}_{T 2}^{2}>\mathscr{U}_{T 3}^{2}$; Q.E.D. In the nonrelativistic limit, the above expression takes the form

$$
\frac{\mathscr{U}_{T 2}^{2}-\mathscr{U}_{T 3}^{2}}{c_{T}^{2}}=\frac{t_{(3)}-t_{(2)}}{\mu},
$$

where $c_{T}^{2}=\tilde{\mu}=\mu / \varrho$ is the standard Lamé value.

Secondly, we may say that purely transverse principal wave fronts are rotational wave fronts whereas purely longitudinal principal wave fronts are dilatational wave

6 This correction to the Lamé moduli is lacking in Carter's work [21] 
fronts. The last statement is obvious from Equation $(3.29)_{1}$. If $\delta u<0$, the wave is compressive and is a rarefaction wave if $\delta u>0$. As to the statement concerning transverse wave fronts, it is sufficient, in order to prove it, to examine the infinitesimal discontinuity of the vorticity spatial four-vector field. The latter is defined by

$$
\Omega^{\mu}=-\frac{1}{2} \eta^{\mu \alpha \beta v}\left(\nabla_{\beta} u_{\alpha}\right) u_{v}=\frac{1}{2} \eta^{\mu \beta \alpha}\left(\stackrel{1}{\nabla}_{\beta} u_{\alpha}\right)
$$

where $\eta^{\mu \beta \alpha} \equiv(-g)^{-1 / 2} \varepsilon^{\mu \beta \alpha \nu} u_{v}, \varepsilon^{\mu \beta \alpha \nu}$ being the four-dimensional alternation symbol. On account of the continuity of $g_{\alpha \beta}$ and $u^{\alpha}$ across $W$ and of the decomposition (2.27), we have

$$
\delta \Omega^{\mu}=\frac{1}{2} \eta^{\mu \beta \alpha} \lambda_{\beta} \delta u_{\perp \alpha}
$$

Hence,

$$
\lambda_{\mu} \delta \Omega^{\mu}=0, \quad S_{\alpha \beta}\left(\delta u_{\perp}^{\alpha}\right) \delta \Omega^{\beta}=0 .
$$

This means that $\delta \boldsymbol{\Omega}$ is orthogonal to $\delta \boldsymbol{u}_{\perp}$ in $H_{(\lambda)}$ and that $\delta \boldsymbol{\Omega}$ is zero if and only if $\delta u_{\perp}^{\beta}$ $=0$. This completes the proof of the statement.

\subsection{Mixed Wave Fronts}

The brief study made in $\S 3.2$ suggests to introduce the following notation to look upon the general case of coupled Equations (3.20) and (3.21) when $\lambda_{\alpha}$ is not an eigenvector of $t_{\alpha \beta}$ :

$$
\begin{aligned}
Q_{\|}\left(\mathscr{U}^{2}, \mathfrak{M}_{0}\right) & \equiv \varrho \mathscr{U}^{2} \bar{F}-(\lambda+2 \mu-3 \bar{T}), \\
Q_{\perp . \beta}^{\alpha}\left(\mathscr{U}^{2}, \mathfrak{M}_{0}\right) & \equiv \varrho \mathscr{U}^{2} \bar{F}_{. \beta}^{\alpha}+\bar{T}_{. \beta}^{\alpha}-\mu S_{. \beta}^{\alpha}, \quad Q_{\perp \alpha \beta}=Q_{\perp \beta \alpha}, \quad Q_{\perp \alpha \beta} \lambda^{\beta}=0, \\
q^{\alpha}\left(\mathscr{U}^{2}, \mathfrak{M}_{0}\right) & \equiv \varrho \mathscr{U}^{2} \bar{F}^{\alpha}+2 \bar{T}^{\alpha}, \quad q^{\alpha} \lambda_{\alpha}=0 .
\end{aligned}
$$

Equations (3.20) and (3.21) read thus

$$
\begin{gathered}
Q_{\perp . \beta}^{\alpha} \delta u_{\perp}^{\beta}+q^{\alpha} \delta u=0, \\
q_{\beta} \delta u_{\perp}^{\beta}+Q_{\|} \delta u=0 .
\end{gathered}
$$

The situation described by these equations can be compared with that met in crystal-like structures (e.g., in [23]). However, the anisotropy, hence the general coupling of the three elastic waves, here is due to the presence of a general initial state of stress. The wave fronts loose their purely transverse or longitudinal character or, in other words, the separation between transverse and longitudinal wave fronts cannot be achieved, eventhough, in certain circumstances, some wave fronts can be called quasi-transverse ${ }^{7}$ or quasi-longitudinal (cf. [23], Chapter 3) waves.

\footnotetext{
7 In agreement with the discussion of $\S 3.2$, this situation occurs when one of the eigenvectors of $t_{\alpha \beta}$ is contained within a cone of axis $\lambda$ and of very accute angle of order $\varepsilon$, if $\varepsilon$ is an infinitesimally small. This defines some notion of neighborhood and of quasi-longitudinality and quasi-transversality. If this is the case, then the values determined in the general case of $\S 3.2$ are valid at the order $\varepsilon$ for $\mathscr{U}$ (however, the transition $\varepsilon \rightarrow 0$ may be singular).
} 
Let $\boldsymbol{e}_{(j)}, j=2,3$, be two unit vectors of $H_{(\lambda)}$ such that their covariant components satisfy the relations

$$
P^{\alpha \beta} e_{(j) \alpha} \lambda_{\beta}=0, \quad e_{(j) \alpha} u^{\alpha}=0, \quad S^{\alpha \beta} e_{(2) \alpha} e_{(3) \beta}=0 .
$$

(j) is a labelling and the tensorial indices are raised and lowered with the help of $S_{\alpha \beta}$. Along with $\boldsymbol{\lambda}$ und $\boldsymbol{u}$, these vectors form a tetrad in curved space-time, whereas $\boldsymbol{e}_{(j)}$ and $\boldsymbol{\lambda}$ form a spatial triad. Let $\delta u_{(j)}$ be nonholonomic components of $\delta \boldsymbol{u}_{\perp}$ on $\left\{\boldsymbol{e}_{(j)} ; j\right.$ $=2,3\}$. Then

$$
\delta u_{\perp}^{\alpha}=\sum_{j=2,3} \delta u_{(j)} e_{(j)}^{\alpha},
$$

and Equations (3.41) and (3.42) can be written as

$$
\begin{aligned}
& \sum_{j=2,3} Q_{\perp k j} \delta u_{(j)}+q_{k} \delta u=0, \quad(k=2,3), \\
& \sum_{j=2,3} q_{j} \delta u_{(j)}+Q_{\|} \delta u=0,
\end{aligned}
$$

where

$$
Q_{\perp k j}=Q_{\perp j k}=e_{(k) \alpha} Q_{\perp . \beta}^{\alpha} e_{(j)}^{\beta}, \quad q_{k}=e_{(k)}^{\alpha} q_{\alpha} .
$$

Equations (3.45) and (3.46) form a system of three equations linear in the unknowns $\delta u$ and $\delta u_{(j)}$. This can be solved for these discontinuities if and only if the corresponding determinant is zero. This yields a cubic equation in $\varrho \mathscr{U}^{2}$ according to the definitions (3.38)-(3.40). That is,

$$
Q_{\|}\left(Q_{\perp 22} Q_{\perp 33}-Q_{\perp 23}^{2}\right)=q_{3}^{2} Q_{\perp 22}+q_{2}^{2} Q_{\perp 33}-2 q_{2} q_{3} Q_{\perp 23} .
$$

For a principal wave the right-hand side of this equation vanishes and we recover the case of §3.2. Otherwise, one must deal with the general Equation (3.48). Let us put it in a form more common in classical problems of acoustics. Define a thirdrank matrix of components $A_{K L}=A_{L K}, K, L=2,3,1$, in such a way that ( $T$ $=$ transpose)

$$
\boldsymbol{A}=\left(\begin{array}{c:c}
\boldsymbol{Q}_{1} & \boldsymbol{q} \\
\hdashline \boldsymbol{q} & Q_{\|}
\end{array}\right),
$$

where the definitions (3.47) have been used. Setting

$\delta \boldsymbol{u}=\left\{\delta u_{L} ; L=2,3,1\right\}=\left(\delta u_{(2)}, \delta u_{(3)}, \delta u\right)-$ a column vector-, Equations (3.45) and (3.46) can be rewritten as

$$
\left[\Gamma_{K L}\left(\mathscr{U}^{2}, \mathfrak{M}_{0}\right)-\varrho \mathscr{U}^{2} \delta_{K L}\right] \delta u_{L}=0, \quad K=2,3,1,
$$

where

$$
\Gamma_{K L} \equiv \bar{F}^{-1} A_{K L}+\varrho \mathscr{U}^{2} \delta_{K L}=\Gamma_{L K}
$$

is Christoffel's acoustic tensor. The reality of the roots $\mathscr{U}$ of the compatibility condition

$$
\underset{3 \times 3}{\operatorname{det}}\left|\Gamma_{K L}-\varrho \mathscr{U}^{2} \delta_{K L}\right|=\operatorname{det}_{3 \times 3}\left|A_{K L}\right|=0,
$$


which is none other than Equation (3.48) written in a different manner, requires that

$$
\Gamma_{K L} N_{K} N_{L}>0
$$

for any vector field $N$ of $\mathbb{R}^{3}$. This condition is not automatically satisfied as a consequence of thermodynamical arguments as is usual in elastodynamics, for $\Gamma_{K L}$ depends on $\mathfrak{M}_{0}$. In the general case (3.52), Equation (3.53) leads to the condition that all principal minors of $A_{K L}$ be positive. Then, in general, there exist three wave fronts that propagate along the same direction but with different speeds and mutually orthogonal polarizations. It can be conjectured that, like in crystal structures, that wave front whose polarization direction is "closest" to the direction of $\lambda$ propagates faster than the other two wave fronts. Thus, for quasi-longitudinal and quasi-transverse waves we shall have $0<\mathscr{U}_{Q T 2}<\mathscr{U}_{Q T 3}<\mathscr{U}_{Q L}<1$. For arbitrarily given $\mathfrak{M}_{0}$ and propagation direction, the checking of the condition (3.53) and the evaluation of the characteristic speeds, and the computation of the rate of growth or decay of the corresponding amplitudes along the rays can be performed only by means of computers, which is not here our purpose. However, a qualitative study of this propagation can be achieved, with the sole help of analytical tools, for principal waves.

\subsection{Propagation along Rays}

The study of the distorsion, growth or decay, that signals-i.e., infinitesimal discontinuities - suffer requires establishing an equation which governs the amplitude of a typical infinitesimal discontinuity along the corresponding ray or bicharacteristic. Given the difficulty met for more complicated cases-e.g., for transverse wave fronts and for a propagation along an arbitrary direction-, we limit the present study to the case of longitudinal principal wave fronts in an initially stressed relativistic elastic body. Of course, the notion of "principalness" in general is purely local. However, it may be global (i.e., for a whole body) in certain circumstances encountered in astrophysics as, for instance, in the case of a body whose natural (i.e., in absence of stresses) shape is a sphere, and that takes the shape of an ellipsoid when acted upon by an initial state of stresses. Then longitudinal principal waves travelling down the principal axes of the ellipsoid remain principal waves in the course of their propagation. In the case of interest here, the wave equation is given by the vanishing of the factor of $\delta u$ in Equation (3.27). This wave equation can also be written in the form (since $L \neq 0$ )

$$
P(l)=H^{\alpha \beta} l_{\alpha} l_{\beta}=0,
$$

where

$$
H^{\alpha \beta} \equiv \varrho \bar{F} u^{\alpha} u^{\beta}-(\lambda+2 \mu-3 \bar{T}) P^{\alpha \beta}=H^{\beta \alpha} .
$$

The characteristics of the wave Equation (3.54) are the hypersurfaces tangent to the second-degree cone $\Sigma$ defined by duality from the symmetrical (nonspatial) tensor $H^{\alpha \beta}$. The contact generatrix of $\Sigma$ with the cone is defined by

$$
N^{\beta}=\frac{1}{2} \partial P(\boldsymbol{l}) / \partial l_{\beta}=H^{\alpha \beta} l_{\alpha} .
$$


The rays or bicharacteristics associated with, and generated by, the wave are the trajectories on $\Sigma$ of the vector field $N^{\beta}$. They are isotropic geodesics [cf. Eq. (3.54)] with respect to the normal hyperbolic (since $\mathscr{U}_{L}<1$ ) metric defined by $H_{\alpha \beta}$. The operator of differentiation along the rays is obviously given by

$$
D_{R} \equiv N^{\beta} \nabla_{\beta}=\varrho \bar{F}\left(u^{\alpha} l_{\alpha}\right) D-(\lambda+2 \mu-3 \bar{T}) l^{\alpha} \frac{1}{D_{\alpha}}
$$

on account of (3.55) and (3.56) and of the definitions of $D$ and $\frac{1}{V_{\alpha}}$.

In order to obtain the propagation equation we take the covariant derivative $\nabla_{\mu}$ of Equations (3.1) through (3.4). That is,

$$
\begin{aligned}
& \left(\nabla_{\mu} u^{\alpha}\right) \nabla_{\alpha} \varrho+u^{\alpha} \nabla_{\mu} \nabla_{\alpha} \varrho+\left(\nabla_{\mu} \varrho\right) \nabla_{\gamma} u^{\gamma}+\varrho \nabla_{\mu} \nabla_{\gamma} u^{\gamma}=0, \\
& \left(\nabla_{\mu} \varrho\right) D \varepsilon+\varrho\left(\nabla_{\mu} u^{\gamma}\right) \nabla_{\gamma} \varepsilon+\varrho u^{\gamma}\left(\nabla_{\mu} \nabla_{\gamma} \varepsilon\right) \\
& \quad-\left(\nabla_{\mu} t^{\alpha \beta}\right) d_{\alpha \beta}-t^{\alpha \beta} \nabla_{\mu} \nabla_{\alpha} u_{\beta}=0, \\
& \left(\nabla_{\mu} \varrho\right) f_{. \beta}^{\alpha} D u^{\beta}+\varrho\left(\nabla_{\mu} f_{. \beta}^{\alpha}\right) D u^{\beta}+\varrho f_{. \beta}^{\alpha}\left(\nabla_{\mu} u^{\gamma}\right) \nabla_{\gamma} u^{\beta} \\
& \quad+\varrho f_{. \beta}^{\alpha} u^{\gamma} \nabla_{\mu} \nabla_{\gamma} u^{\beta}-\left(\nabla_{\mu} P_{. \gamma}^{\alpha}\right) \nabla_{\beta} t^{\gamma \beta}-P_{. \gamma}^{\alpha} \nabla_{\mu} \nabla_{\beta} t^{\gamma \beta}=0,
\end{aligned}
$$

and

$$
\begin{aligned}
& \left(\nabla_{\mu} u^{\gamma}\right)\left(\nabla_{\gamma} t_{\alpha \beta}\right)_{\perp}+u^{\gamma} \nabla_{\mu}\left(\nabla_{\gamma} t_{\alpha \beta}\right)_{\perp}+\left(\nabla_{\mu} t_{\gamma \beta}\right) \frac{1}{\nabla} u^{\gamma} \\
& \quad+t_{\gamma \beta} \nabla_{\mu} \frac{1}{\nabla_{\alpha}} u^{\gamma}+\left(\nabla_{\mu} t_{\alpha \gamma}\right) \frac{1}{\nabla_{\beta}} u^{\gamma}+t_{\alpha \gamma} \nabla_{\mu} \frac{1}{\nabla_{\beta}} u^{\gamma} \\
& \quad+\left(\nabla_{\mu} t_{\alpha \beta}\right) \nabla_{\gamma} u^{\gamma}+t_{\alpha \beta}\left(\nabla_{\mu} \nabla_{\gamma} u^{\gamma}\right) \\
& =\left(\nabla_{\mu} \varrho\right)\left[\bar{\lambda}_{\gamma_{\gamma}} P_{\alpha \beta}+2 \tilde{\mu} d_{\alpha \beta}\right]+\lambda\left(\nabla_{\mu} \nabla_{\gamma} u^{\gamma}\right) P_{\alpha \beta} \\
& \quad+\lambda\left(\nabla_{\gamma} u^{\gamma}\right) \nabla_{\mu}\left(u_{\alpha} u_{\beta}\right)+\mu\left(\nabla_{\mu} \bar{\nabla}_{\alpha} u_{\beta}+\nabla_{\mu} \bar{\nabla}_{\beta} u_{\alpha}\right) .
\end{aligned}
$$

We use the hypotheses set forth in $\S 2.4$. Moreover, following Lichnerowicz ([11], pp. 100 and 108), we know that there exist generalized functions $\bar{u}^{\lambda}, \bar{\varepsilon}$, and $\bar{\varrho}$ such that

$$
\begin{aligned}
\tilde{\delta}\left[\nabla_{\alpha} \nabla_{\beta} \varrho \rrbracket\right. & =\left(\nabla_{\alpha} l_{\beta}\right) \delta \varrho+l_{\alpha} \nabla_{\beta} \delta \varrho+l_{\beta} \nabla_{\alpha} \delta \varrho+l_{\alpha} l_{\beta} \bar{Q}, \\
\tilde{\delta}\left[\nabla_{\alpha} \nabla_{\beta} u^{\lambda}\right] & =\left(\nabla_{\alpha} l_{\beta}\right) \delta u^{\lambda}+l_{\alpha} \nabla_{\beta} \delta u^{\lambda}+l_{\beta} \nabla_{\alpha} \delta u^{\lambda}+l_{\alpha} l_{\beta} \bar{u}^{\lambda},
\end{aligned}
$$

and

$$
\bar{\delta}\left[\nabla_{\alpha} \nabla_{\beta} \varepsilon\right]=l_{\alpha} l_{\beta} \bar{\varepsilon}
$$

Consider Equation (3.58) on both sides of the wave front $W$ and substract the two resulting expressions. We have thus

$$
u^{\alpha} \tilde{\delta}\left[\nabla_{\mu} \nabla_{\alpha} \varrho\right]+\varrho \bar{\delta}\left[\nabla_{\mu} \nabla_{\gamma} u^{\gamma}\right]+\bar{\delta}\left[\left(\nabla_{\mu} u^{\alpha}\right) \nabla_{\alpha} \varrho\right]+\bar{\delta}\left[\left(\nabla_{\mu} \varrho\right) \nabla_{\gamma} u^{\gamma}\right]=0
$$

But

$$
\bar{\delta}\left[\left(\nabla_{\mu} u^{\alpha}\right) \nabla_{\alpha} \varrho\right]=\left\{\nabla_{\mu} u^{\alpha}\right\}^{+} \bar{\delta}\left[\nabla_{\alpha} \varrho\right]+\left\{\nabla_{\alpha} \varrho\right\}^{-} \bar{\delta}\left[\nabla_{\mu} u^{\alpha} \rrbracket\right.
$$


The right-hand side of the last equation is linear in both $\delta \varrho$ and $\delta u^{\alpha}$, hence is linear in $\delta u^{\alpha}$ on account of Equation (3.5), thus linear in $\delta u$ for a longitudinal principal wave front. The same comment applies to the last contribution in the left-hand side of (3.65). This equation can be written thus

$$
u^{\alpha} \bar{\delta}\left[\nabla_{\mu} \nabla_{\alpha} \varrho \rrbracket+\varrho \bar{\delta} \llbracket\left[\nabla_{\mu} \nabla_{\gamma} u^{\gamma} \rrbracket \cong 0(\delta u)\right.\right.
$$

where $\cong 0(\delta u)$ means modulo terms proportional to $\delta u$.

Consider now Equation (3.59) and apply the same procedure. After inner product of the resulting jump relation with $u^{\mu}$, we obtain

$$
\varrho u^{\gamma} u^{\mu} \bar{\delta}\left[\nabla_{\mu} \nabla_{\gamma} \varepsilon\right]-t^{\alpha \beta} u^{\mu} \bar{\delta}\left[\nabla_{\mu} \nabla_{\beta} u_{\alpha}\right] \cong 0(\delta u, \delta \varepsilon, \delta \boldsymbol{t}) \text {. }
$$

The right-hand side of this equation can be replaced by $0(\delta u)$ on account of Equations (3.6) and (3.9) through (3.13) - written down for longitudinal principal waves.

Next, consider Equation (3.60). We get thus

$$
\varrho f_{. \beta}^{\alpha} u^{\gamma} \bar{\delta} \llbracket \nabla_{\mu} \nabla_{\gamma} u^{\beta} \rrbracket-P_{. \gamma}^{\alpha} \bar{\delta} \llbracket\left[\nabla_{\mu} \frac{1}{\nabla_{\dot{\beta}}} \nu^{\gamma \beta} \rrbracket \cong 0(\delta u, \delta \boldsymbol{t})\right. \text {. }
$$

The right-hand side of this equation can be replaced by $O(\delta u)$ on account of Equations (3.9) through (3.13)-written down for longitudinal principal waves. Finally, consider Equation (3.61). By the same reasoning and after rearranging the indices, we obtain

$$
\begin{aligned}
& u^{\gamma} \bar{\delta} \llbracket \nabla_{\gamma} \nabla_{\beta} t^{\alpha \beta} \rrbracket-\lambda P^{\alpha \beta} \bar{\delta} \llbracket\left[\nabla_{\beta} \nabla_{\gamma} u^{\gamma} \rrbracket-\mu \bar{\delta} \llbracket \nabla_{\beta} \nabla^{\alpha} u^{\beta}+\nabla_{\beta} \nabla^{\beta} u^{\alpha}\right]_{\perp} \\
& \quad+t_{. \gamma}^{\alpha} \bar{\delta} \llbracket \nabla_{\beta} \nabla^{\beta} u^{\gamma} \rrbracket+t_{\gamma}^{\beta} \bar{\delta}\left[\nabla_{\beta} \nabla^{\alpha} u^{\gamma} \rrbracket_{\perp}+t^{\alpha \beta} \bar{\delta}\left[\nabla_{\beta} \nabla_{\gamma} u^{\gamma} \rrbracket\right.\right. \\
& \cong 0\left(\delta u, \delta u^{2}\right),
\end{aligned}
$$

where $\cong 0\left(\delta u, \delta u^{2}\right)$ means modulo terms proportional to $\delta u$ and to $(\delta u)^{2}$. Remark that the factor of $(\delta u)^{2}$ comes from the nonlinearity of the right-hand side of Equation (3.4) which results from the spatial character (hence the use of the spatial projector which is quadratic in the four-velocity). Now contract Equation (3.69) with $u^{\mu}$ and eliminate $u^{\mu} \bar{\delta}\left[\nabla_{\mu} \nabla_{\beta} t^{\gamma \beta}\right]$ from the resulting equation with the help of Equation (3.70). Rearranging the indices, we have thus

$$
\begin{aligned}
& \left\{\varrho f_{. \beta}^{\alpha} u^{\gamma} u^{\mu}-\lambda P^{\alpha \mu} P_{\beta}^{\cdot \gamma}-\mu\left(P^{\alpha \gamma} P_{\beta}^{\mu}+P_{. \beta}^{\alpha} P^{\gamma \mu}\right)\right. \\
& \left.\quad+t_{. \beta}^{\alpha} P^{\mu \gamma}+t_{\beta}^{\cdot \mu} P^{\alpha \gamma}+t^{\alpha \mu} P^{\gamma \beta}\right\} \bar{\delta} \llbracket \nabla_{\mu} \nabla_{\gamma} u^{\beta} \rrbracket \\
& \cong 0\left(\delta u, \delta u^{2}\right) .
\end{aligned}
$$

Now use an expression of the type (3.63) to express $\bar{\delta}\left[\nabla_{\mu} \nabla_{\gamma} u^{\beta}\right]$ and consider the case of longitudinal principal waves for which $\delta u^{\beta}=L P^{\beta \varepsilon} l_{\varepsilon} \delta u$. The term in $\bar{u}^{\beta}$ disappears on account of Equation (3.54). The terms in $\nabla_{\alpha} l_{\beta}$ are proportional to $\delta u$ and involves the initial stresses and the initial solution $\mathfrak{M}_{0}$. They are thus incorporated in the right-hand side and it remains, on account of the symmetry of $H^{\alpha \beta}$,

$$
H^{\alpha \beta} l_{\alpha} \nabla_{\beta} \delta u \cong 0\left(\delta u, \delta u^{2}\right) .
$$

This is, on account of (3.56) and (3.57), and using a symbolic notation,

$$
D_{R} \delta u-A\left(\mathscr{G}_{(2)}^{W}, \mathfrak{M}_{0}\right) \delta u-B\left(\mathscr{U}_{L}, \mathfrak{M}_{0}\right) \delta u^{2}=0,
$$


where $\mathscr{G}_{(2)}^{W}$ means the geometry of the wave front at the second order, e.g., the curvature which intervenes via $\nabla_{\alpha} l_{\beta}$ (cf. [19]). $A$ and $B$ are two scalar coefficients which vary along the rays for nonuniform initial states and of which the sign plays a fundamental role. In particular, if $\mathfrak{M}_{0}$ is such that, initially, $\nabla_{\alpha} u^{\beta}=0$, then $B=0$ and it can be shown using the results of [19] that $A$ reduces to the mean curvature $\Omega$ of the spatial part of $W$. Then Equation (3.73) takes the form $D_{R} \delta u=\Omega \delta u$. For a spherical front $\Omega=(-1 / R)$ with $R>0$ and if, furthermore, the wave front is compressive, i.e., $\delta u<0$, then the above reduced equation shows that, since $\mathscr{U}$ is constant, the $W$-surfaces at different times form a system of parallel surfaces, and the wave is damped out. However, for the general case described by the propagation Equation (3.73), if $\lambda$, $\mu$, and $\mathfrak{M}_{0}$ are such that $B<0$, then, for a compressive wave, Equation (3.73) shows that a compressive wave terminates into a "shock" " while it is damped out if $B>0$. The converse is true for a rarefaction wave. Of course, the requirement that only a compressive wave can terminate into a shock imposes a supplementary restriction on the material constants and on the initial state. The above result (3.73), which constitutes the fundamental property of rays associated with longitudinal principal wave fronts, clearly is of great importance for astrophysical problems involving discontinuities in massive stellar objects of which the interior can be considered as elastic in accord with our definition of relativistic elasticity.

To conclude this section, it can be remarked that Equation (3.67) can be used to show that the infinitesimal discontinuity $\delta \varrho$ propagates in accordance with an equation similar to (3.73). As to Equation (3.68), it yields $\bar{\varepsilon}$ as a function of $\mathscr{U}_{L}$ and of the geometry $\mathscr{G}_{(2)}^{W}$, as a linear expression in $\delta u$, and an expression which involves $\nabla_{\alpha} \delta u$ and is linear in the initial value of the stresses. In absence of such stresses, we have therefore: $\bar{\varepsilon} \cong 0(\delta u)$.

\section{A. Hypoelasticity Deduced from Neo-Hookean Elasticity}

\subsection{Strains}

Two basic ingredients are missing in the theory of elasticity sketched out in $\S 2.3$ : the notion of strain and a thermodynamical argument. These are related to one another as will be shown shortly. Let $G_{K L}=G_{L K},(K, L=1,2,3)$, be the time-invariant local reference metric on the material three-dimensional manifold $\mathscr{M}^{3}$. A material body is an open, bounded, simply connected region of $\mathscr{M}^{3}$. Then let $\mathbb{B}$ be Pfaffian forms (cf. [25]) - whose components $B_{. \alpha}^{K}, K=1,2,3$, are spatial four-vector fields on $V^{4}$ - that describe the, in general, anelastic deformation of the body. The image of $G_{K L}$ by $B$ defines a symmetric spatial covariant tensor field on $V^{4}$ :

$$
G_{\alpha \beta}=G_{K L} B_{. \alpha}^{K} B_{. \beta}^{L}=G_{\beta \alpha}, \quad G_{\alpha \beta} u^{\beta}=0 .
$$

For a purely elastic deformation, we have [25]

$$
B_{. \alpha}^{K} \equiv \partial X^{K} / \partial x^{\alpha}
$$

8 Compare Whitham [24], p. 133 
if $X^{K}=\mathscr{X}^{K}\left(x^{\alpha}\right)$ represents the canonical differentiable projection $\mathscr{P}: V^{4} \rightarrow \mathscr{M}^{3}$. We have $D X^{K}=0$ in virtue of the independence of $X^{K}$ on the proper time. $G_{K L}$ being time-invariant, it is then deduced that $\underset{u}{\mathfrak{f}} G_{\alpha \beta}=0$ (see, e.g., [5]). The relativistic Euler strain tensor being defined by

$$
\mathscr{E}_{\alpha \beta} \equiv \frac{1}{2}\left(P_{\alpha \beta}-G_{\alpha \beta}\right)=\mathscr{E}_{\beta \alpha}, \quad \mathscr{E}_{\alpha \beta} u^{\beta}=0,
$$

we deduce from (2.3) that

$$
\mathfrak{f}_{u} \mathscr{E}_{\alpha \beta}=d_{\alpha \beta} \text {. }
$$

\subsection{Thermodynamical Argument}

It can be shown that exact constitutive equations for a nonlinear elastic material in relativistic motion are given in function of the internal energy density $\varepsilon\left(\mathscr{E}_{\alpha \beta}, \eta\right)$ by [26]

$$
t^{\alpha \beta}=\varrho\left(\frac{\partial \varepsilon}{\partial \mathscr{E}_{\ddot{\alpha \beta}}}-2 \mathscr{E}_{\cdot \nu}\left(\frac{\partial \varepsilon}{\partial \mathscr{E}_{\dot{\beta}) \dot{\gamma}}}\right),\right.
$$

and

$$
\theta=\frac{\partial \varepsilon}{\partial \eta}
$$

where $\eta$ and $\theta$ are the entropy per unit of proper mass and the proper thermodynamical temperature, respectively. Equation (4.5) is the relativistic version of Murnaghan's formulation of classical nonlinear elasticity (cf. [27]). Furthermore, the condition of objectivity referred to in $\S 3.2$ imposes that $\varepsilon$ be an isotropic function of its tensorial argument $\mathscr{E}_{\alpha \beta}$, hence depend on $\mathscr{E}_{\alpha \beta}$ only via its three principal invariants $I_{(j)} \equiv \operatorname{tr} \mathscr{E}^{j}, j=1,2,3$, where $\operatorname{tr}=$ trace. Then Equation (2.11) can be replaced by the condition of adiabaticity

$$
\varrho \theta D \eta=0 \text {. }
$$

If $\mathscr{E}_{\alpha \beta}$ satisfies the condition

$$
\left[\operatorname{tr} \mathscr{E}^{2}\right]^{1 / 2} \ll 1,
$$

then we say that the relativistic deformation is infinitesimally small, and we can replace Equation $(4.5)$ and the general function $\varepsilon\left(I_{(j)}, \eta\right)$ by the following approximations :

$$
t^{\alpha \beta}=\varrho \partial \varepsilon / \partial \mathscr{E}_{\alpha \beta},
$$

and

$$
\varepsilon=\frac{1}{2} \tilde{\lambda} I_{(1)}^{2}+\tilde{\mu} I_{(2)}-\tilde{\kappa} I_{(1)} \eta+\theta_{0} \eta+\frac{\gamma}{2} \eta^{2}
$$

where, $\tilde{\lambda}, \tilde{\mu}, \tilde{\kappa}, \gamma$, and $\theta_{0}$ are pure constants. It follows from (4.9) and (4.6) that

$$
t_{\alpha \beta}=\varrho\left[\left(\tilde{\lambda} \mathscr{E}_{. \gamma}^{\gamma}-\tilde{\kappa} \eta\right) P_{\alpha \beta}+2 \tilde{\mu} \mathscr{E}_{\alpha \beta}\right] \text {, }
$$


and

$$
\theta=\theta_{0}-\tilde{\kappa} \mathscr{E}{ }_{. \gamma}^{\gamma}+\gamma \eta
$$

Thermodynamical considerations require that (compare [28], p. 28)

$$
\left.\begin{array}{l}
\tilde{\mu}>0, \quad \Lambda \equiv 3 \tilde{\lambda}+2 \tilde{\mu}>0, \quad \gamma>0, \quad \theta_{0} \geqq 0, \\
\theta>0, \quad \gamma>\left(\tilde{\kappa}^{2} / \Lambda\right) .
\end{array}\right\}
$$

$\tilde{\kappa}$ is positive or negative depending on whether the solid expands or contracts on heating.

We call Neo-Hookean bodies relativistic elastic bodies whose constitutive equations are given by Equations (4.10) through (4.12). In accord with previous comments (cf. [5], Appendix), stress-strain relations of the type (4.11) are said to be quasi-linear for, although they are explicitly linear in $\mathscr{E}_{\alpha \beta}$, they also involve the strains via the factor $\varrho$. Furthermore, Equation (4.11) have the following important property. Take the invariant derivative $D$ of both sides of Equation (4.11), project spatially the resulting equation while taking account of the fact that $\left(D P_{\alpha \beta}\right)_{\perp} \equiv 0$, and reintroduce Lie derivatives on account of Equations (4.4) and (2.4). Then, with the notation (2.16), we have

$$
{ }^{*} t_{\alpha \beta}=\varrho\left[\tilde{\lambda}\left(d_{\cdot \gamma}^{\gamma}-2 \mathscr{E}^{\mu \nu} d_{\mu \nu}\right)-\tilde{\kappa} D \eta\right] P_{\alpha \beta}+2 \varrho\left(\tilde{\mu}+\tilde{\lambda} \mathscr{E}_{\cdot \mu}^{\mu}-\tilde{\kappa} \eta\right) d_{\alpha \beta} .
$$

$\mathscr{E}_{\alpha \beta}$ is expressed by inverting (4.11) on account of the fact that $P_{. \alpha}^{\alpha}=3$. That is,

$$
\mathscr{E}_{\alpha \beta}=\frac{1}{2 \varrho \tilde{\mu}}\left[t_{\alpha \beta}-\left(\frac{\tilde{\lambda} t_{. \gamma}^{\gamma}-2 \varrho \tilde{\mu} \tilde{\kappa} \eta}{\Lambda}\right) P_{\alpha \beta}\right],
$$

and

$$
\mathscr{E}_{\cdot \mu}^{\mu}=\left(t_{. \gamma}^{\gamma}+3 \varrho \tilde{\kappa} \eta\right) / \varrho \Lambda .
$$

Substituting these two results in Equation (4.14), we are led to

$$
\begin{aligned}
{ }^{*} t_{\alpha \beta}= & \varrho\left\{\tilde{\lambda}[1-(2 \tilde{\kappa} \eta / \Lambda)] d_{. \gamma}^{\gamma}-\tilde{\kappa} D \eta\right\} P_{\alpha \beta} \\
& +(\tilde{\lambda} / \tilde{\mu})\left[\left(\tilde{\lambda} t_{. \gamma}^{\gamma} d_{\cdot \mu}^{\mu}\right) /(\varrho \Lambda)-t^{\mu \nu} d_{\mu \nu}\right] P_{\alpha \beta} \\
& +2 \varrho \tilde{\mu}\left[1-(2 \tilde{\kappa} \eta / \Lambda)+\left(\tilde{\lambda} t_{. \gamma}^{\gamma}\right) /(\varrho \tilde{\mu} \Lambda)\right] d_{\alpha \beta} .
\end{aligned}
$$

Of course, Equation (4.7) implies that $D \eta=0$. Then Equation (4.17) is of the same type as a constitutive equation for a hypoelastic body of first order, that is, Equation (2.15), with $\mathscr{L}_{\alpha \beta}^{. \mu \nu}$ given by (2.19) and with the following substitutions:

$$
\left.\begin{array}{l}
\tilde{\lambda} \rightarrow \tilde{\lambda}[1-(2 \tilde{\kappa} \eta / \Lambda)], \quad \varrho \tilde{\lambda}^{\prime} \rightarrow \tilde{\lambda}^{2} / \tilde{\mu} \Lambda, \quad \tilde{\mu} \rightarrow \tilde{\mu}[1-(2 \tilde{\kappa} \eta / \Lambda)], \\
\varrho \tilde{\mu}^{\prime} \rightarrow \tilde{\lambda} / \Lambda, \quad \varrho \tilde{\alpha} \rightarrow(-\tilde{\lambda} / \tilde{\mu}), \quad \tilde{\beta} \rightarrow 0, \quad \tilde{\gamma} \rightarrow 0 .
\end{array}\right\}
$$

The property just placed in evidence shows that two directions can be followed for studying infinitesimal discontinuities in relativistic elastic bodies whose constitutive equations are derivable from the potential (4.10) and within the framework of infinitesimally small deformations. The first one is a direct treatment, in which case Equation (4.4) will be used as the compatibility condition whereas the constitutive Equation (4.11) must be substituted for in the motion Equation (2.12). This is briefly examined in the next paragraph. The second direction makes use directly of 
Equation (3.7), in which case it is Equation (4.17) that is used as a compatibility condition. This second possibility is none other than a special case of the treatment of infinitesimal discontinuities in hypoelastic solids of the first order. This will be examined in Section 5. In both cases, however, the following two remarks hold true. Firstly, $\varepsilon$ and its derivatives being assumed continuous across the wave front $W$, Equation (4.7) implies that

$$
\mathscr{U} \delta \eta=0 .
$$

Thus $\delta \eta \neq 0$ implies $\mathscr{U}=0$, and material wave fronts $(\mathscr{U}=0)$ can also be called entropy wave fronts. Secondly, on account of Equation (4.11), the principal directions of both $t_{\alpha \beta}$ and $\mathscr{E}_{\alpha \beta}$ coincide $^{9}$, the corresponding eigenvalues being related in a simple manner.

\subsection{Direct Treatment of Infinitesimal Discontinuities in Neo-Hookean Elasticity}

We indicate only the great lines of the derivations. Substituting (4.11) into Equation (3.3), we obtain

$$
\varrho f_{. \beta}^{\alpha} D u^{\beta}-\left[\left(\stackrel{\perp}{\nabla}_{\beta} \varrho\right) \varrho^{-1} t^{\alpha \beta}+\varrho \tilde{\lambda}\left(\stackrel{\perp}{\nabla}_{\beta}^{\mathscr{E}_{. \mu}^{\mu}}\right) P^{\alpha \beta}-\varrho \tilde{\kappa}\left(\stackrel{\perp}{\nabla}_{\beta} \eta\right) P^{\alpha \beta}+2 \varrho \tilde{\mu}\left(\stackrel{\perp}{\nabla}_{\beta} \mathscr{E}^{\alpha \beta}\right)_{\perp}\right]=0 .
$$

Taking the infinitesimal discontinuity of Equation (4.4), with $\mathscr{U} \neq 0$, and of Equation (4.20), we get

$$
\left(\delta \mathscr{E}_{\alpha \beta}\right)_{\perp}=\mathscr{U}^{-1}\left[\frac{1}{2}\left(\lambda_{\alpha} \delta u_{\beta}+\lambda_{\beta} \delta u_{\alpha}\right)-\mathscr{E}_{\gamma \beta} \lambda_{\alpha} \delta u^{\gamma}-\mathscr{E}_{\alpha \gamma} \lambda_{\beta} \delta u^{\gamma}\right],
$$

and

$$
\varrho f_{. \beta}^{\alpha} \mathscr{U} \delta u^{\beta}-\varrho^{-1} t^{\alpha \beta} \lambda_{\beta} \delta_{\varrho}-\varrho \tilde{\lambda} \lambda^{\alpha}\left(\delta \mathscr{E}_{. \mu}^{\mu}\right)+\varrho \tilde{\kappa} \lambda^{\alpha} \delta \eta-2 \varrho \tilde{\mu} \lambda_{\beta}\left(\delta \mathscr{E}^{\alpha \beta}\right)_{\perp}=0 .
$$

Taking account of the fact that $\delta \eta=0$, substituting from Equations (3.5) and (4.21) into Equation (4.22), introducing the decomposition of $\mathscr{E}_{\alpha \beta}$ [compare (2.31)] by

$$
\mathscr{E}_{\alpha \beta}=\bar{E} \lambda_{\alpha} \lambda_{\beta}+2 \bar{E}_{(\alpha \lambda \beta)}+\bar{E}_{\alpha \beta},
$$

using the decompositions (2.27), (2.30), and (2.32), and projecting the equation thus deduced from (4.22) in the direction of $\lambda$ and onto $H_{(\lambda)}$, we obtain the following system

$$
\begin{aligned}
& \left(\varrho \mathscr{U}^{2} \bar{F}_{. \beta}^{\alpha}-\mu S_{. \beta}^{\alpha}+2 \mu \bar{E}_{, \beta}^{\alpha}\right) \delta u_{\perp}^{\beta}+\left(\varrho \mathscr{U}^{2} \bar{F}^{\alpha}+\bar{T}^{\alpha}+2 \mu \bar{E}^{\alpha}\right) \delta u=0, \\
& \left(\varrho \mathcal{U}^{2} \bar{F}_{\beta}+4 \mu \bar{E}_{\beta}\right) \delta u_{\perp}^{\beta}+\left[\varrho \mathcal{U}^{2} \bar{F}+\bar{T}-(\lambda+2 \mu)(1-2 \bar{E})\right] \delta u=0 .
\end{aligned}
$$

From Equations (2.30), (4.23), and (4.11) - where we set $\tilde{\kappa}=0$ without much loss of generality-, we deduce that

$$
\begin{aligned}
& \bar{E}_{\beta}=\bar{T}_{\beta} / 2 \mu, \\
& \bar{E}=(2 \mu)^{-1}\left[\bar{T}-\left(\lambda t_{. \gamma}^{\gamma} / \varrho \Lambda\right)\right], \\
& \bar{E}_{. \beta}^{\alpha}=(2 \mu)^{-1}\left[\bar{T}_{. \beta}^{\alpha}-\left(\lambda t_{. \gamma}^{\gamma} / \varrho \Lambda\right) S_{. \beta}^{\alpha}\right] .
\end{aligned}
$$

9 In fact, this property holds good also for the general relationship (4.5) and is due ultimately to the isotropy of the energy density $\varepsilon$ as a function of $\mathscr{E}_{\alpha \beta}$ 
The elements of decomposition of $f_{. \beta}^{\alpha}$ can also be expressed entirely in terms of the relativistic stress via Equations (2.32), (4.15), and (4.16). Then we can rewrite Equations (4.24) and (4.25) in the form of Equations (3.41) and (3.42), but with

$$
\begin{aligned}
& Q_{\|} \equiv \varrho \mathscr{U}^{2} \bar{F}-\left\{\lambda+2 \mu-3 \bar{T}+(\lambda / \mu)\left[\left(\frac{\lambda+2 \mu}{\varrho \Lambda}\right) t_{\cdot \nu}^{\gamma}-\bar{T}\right]\right\}, \\
& q^{\alpha} \equiv \varrho \mathscr{U}^{2} \bar{F}^{\alpha}+2 \bar{T}^{\alpha},
\end{aligned}
$$

and

$$
Q_{\perp . \beta}^{\alpha} \equiv \varrho \mathscr{U}^{2} \bar{F}_{. \beta}^{\alpha}-\left(\mu+\frac{\lambda t_{. \gamma}^{\gamma}}{\varrho \Lambda}\right) S_{. \beta}^{\alpha}+\bar{T}_{. \beta}^{\alpha} .
$$

We see that the differences with the case examined in Section 3 are not drastic. In particular, we have uncoupling for principal wave fronts since, then

$$
\bar{T}_{\alpha}=\bar{E}_{\alpha}=\bar{F}_{\alpha}=0 \text {. }
$$

In this case a computation analogous to that made in Section 3 for a general initial state of stresses yields the following three speeds:

for longitudinal principal waves:

$$
\mathscr{U}_{L 1}^{2}=(\varrho \bar{F})^{-1}\left\{\lambda+2 \mu-3 t_{\|}+(\lambda / \mu)\left[\left(\frac{\lambda+2 \mu}{\varrho \Lambda}\right)\left(t_{(2)}+t_{(3)}\right)-\left(\frac{2 \lambda}{\varrho \Lambda}\right) t_{\| 1}\right]\right\} ;
$$

for transverse principal waves:

$$
\mathscr{U}_{T 2}^{2}=\left[\varrho(1+\varepsilon)-t_{(2)}\right]^{-1}\left[\mu-t_{(2)}\left(1-\frac{\lambda}{\varrho \Lambda}\right)+\frac{\lambda}{\varrho \Lambda}\left(t_{\|}+t_{(3)}\right)\right],
$$

and

$$
\mathscr{U}_{T 3}^{2}=\left[\varrho(1+\varepsilon)-t_{(3)}\right]^{-1}\left[\mu-t_{(3)}\left(1-\frac{\lambda}{\varrho \Lambda}\right)+\frac{\lambda}{\varrho \Lambda}\left(t_{\|}+t_{(2)}\right)\right] .
$$

The condition of reality of the wave speeds obviously imposes restrictions on the coefficients $\lambda$ and $\mu$ and on the initial state of stress. If the initial state is a state of hydrostatic pressure $p_{0}$, then a simple calculation shows that the apparent elasticities in Equation (3.37) are now given by

$$
\bar{\mu}=\mu+p_{0}\left[\frac{1}{1+(3 \lambda / 2 \mu)}\right], \quad \bar{\lambda}=\lambda+p_{0}\left[\frac{1+(4 \lambda / \mu)}{1+(3 \lambda / 2 \mu)}\right] .
$$

The remarks made in $\S 3.3$ are essentially unchanged on account of the form of the system formed from the expressions (4.29) through (4.31). The same holds true of the qualitative results of $\S 3.4$, the formation of shocks, i.e., the growth to infinity in a finite time of the amplitude, still being possible for adequate circumstances.

\section{Imfinimitesimal Discontinuities in Rellativistic Hypoelastic Bodies of First Order}

In this case the derivation of Equations (3.5) - (3.7) is left unchanged. It remains to account for a compatibility condition which is obtained by taking the infinitesimal discontinuity of Equation (2.15) on account of the specific expression (2.19). We 
have thus

$$
\begin{aligned}
\mathscr{U}( & \left.\delta t_{\alpha \beta}\right)_{\perp}+\lambda_{\beta} t_{\alpha \mu} \delta u^{\mu}+\lambda_{\alpha} t_{\beta \mu} \delta u^{\mu}+t_{\alpha \beta} \delta u \\
= & \left(\lambda+\lambda^{\prime} t_{. \gamma}^{\gamma}\right) \delta u P_{\alpha \beta}+\left(\mu+\mu^{\prime} t_{\cdot \gamma}^{\gamma}\right)\left(\lambda_{\alpha} \delta u_{\beta}+\lambda_{\beta} \delta u_{\alpha}\right) \\
& +\frac{1}{2} \alpha t^{\mu \nu}\left(\lambda_{\mu} \delta u_{v}+\lambda_{v} \delta u_{\mu}\right) P_{\alpha \beta}+\beta t_{\alpha \beta} \delta u \\
& +\gamma\left[t_{\beta}^{. \mu}\left(\lambda_{\mu} \delta u_{\alpha}+\lambda_{\alpha} \delta \mu_{\mu}\right)+t_{\alpha}^{\mu}\left(\lambda_{\mu} \delta u_{\beta}+\lambda_{\beta} \delta u_{\mu}\right)\right],
\end{aligned}
$$

with obvious definitions for $\lambda^{\prime}, \mu^{\prime}, \alpha, \beta$, and $\gamma$. Decomposing this equation along $\lambda$ and onto $H_{(\lambda)}$, we obtain

$$
\begin{aligned}
\mathscr{U} \delta \tau+ & 2 \bar{T}_{\alpha}\left(1-\frac{\alpha}{2}-\gamma\right) \delta u_{\perp}^{\alpha}+ \\
+ & \left\{(3-\beta-\alpha-4 \gamma) \bar{T}-\left[\lambda+2 \mu+\left(\lambda^{\prime}+2 \mu^{\prime}\right) t_{. \gamma}^{\gamma}\right]\right\} \delta u=0, \\
\mathscr{U} \delta \tau^{\alpha}- & {\left[\left(\mu+\mu^{\prime} t_{. \gamma}^{\gamma}+\gamma \bar{T}\right) S_{. \beta}^{\alpha}-\bar{T}_{. \beta}^{\alpha}(1-\gamma)\right] \delta u_{\perp}^{\beta} } \\
+ & {[2(1-\gamma)-\beta] \bar{T}^{\alpha} \delta u=0, } \\
\mathscr{U} \delta \tau^{\alpha \beta}= & {\left[\left(\lambda+\lambda^{\prime} t_{. \gamma}^{\gamma}+\alpha \bar{T}\right] S^{\alpha \beta}-(1-\beta) \bar{T}^{\alpha \beta}\right] \delta u } \\
& +\left[\alpha S^{\alpha \beta} \bar{T}_{\mu}+\gamma \bar{T}^{\beta} S_{. \mu}^{\alpha}+\gamma \bar{T}^{\alpha} S_{. \mu}^{\beta}\right] \delta u_{\perp}^{\mu} .
\end{aligned}
$$

Eliminating now $\delta \tau$ and $\delta \tau^{\alpha}$ between Equations (3.9), (3.10), (5.2), and (5.3), we are led to the following linear system:

$$
\begin{aligned}
& {\left[\varrho \mathscr{U}^{2} \bar{F}_{\beta}+2 \bar{T}_{\beta}\left(1-\gamma-\frac{\alpha}{2}\right)\right] \delta u_{\perp}^{\beta}} \\
& +\left\{\varrho \mathscr{U}^{2} \bar{F}-\left[\lambda+2 \mu+\left(\lambda^{\prime}+2 \mu^{\prime}\right) t_{. \gamma}^{\gamma}\right]+(3-\beta-\alpha-4 \gamma) \bar{T}\right\} \delta u=0, \\
& {\left[\varrho \mathscr{U}^{2} \bar{F}_{. \beta}^{\alpha}-\left(\mu+\mu^{\prime} t_{. \gamma}^{\gamma}+\gamma \bar{T}\right) S_{. \beta}^{\alpha}+(1-\gamma) \bar{T}_{. \beta}^{\alpha}\right] \delta u_{\perp}^{\beta}} \\
& +\left\{\varrho \mathscr{U}^{2} \bar{F}+[2(1-\gamma)-\beta] \bar{T}^{\alpha}\right\} \delta u=0 .
\end{aligned}
$$

For principal wave fronts, Equation (4.32) holds good and the system (5.5) and (5.6) uncouples. Similarly to what was done in $\S 3.2$, we obtain the following wave speeds for a general initial state of stress:

for longitudinal principal waves:

$$
\begin{aligned}
\mathscr{U}_{L 1}^{2}= & (\varrho \bar{F})^{-1}\left[\lambda+2 \mu-\left(3-\beta-\alpha-4 \gamma-\lambda^{\prime}-2 \mu^{\prime}\right) t_{\|}\right. \\
& \left.+\left(\lambda^{\prime}+2 \mu^{\prime}\right)\left(t_{(2)}+t_{(3)}\right)\right] ;
\end{aligned}
$$

for transverse principal waves:

$$
\mathscr{U}_{T 2}^{2}=\left(\varrho \bar{F}_{.2}^{2}\right)^{-1}\left[\mu-t_{(2)}\left(1-\gamma-\mu^{\prime}\right)+\left(\gamma+\mu^{\prime}\right) t_{\| 1}+\mu^{\prime} t_{(3)}\right],
$$

and

$$
\mathscr{U}_{T 3}^{2}=\left(\varrho \bar{F}_{.3}^{3}\right)^{-1}\left[\mu-t_{(3)}\left(1-\gamma-\mu^{\prime}\right)+\left(\gamma+\mu^{\prime}\right) t_{||}+\mu^{\prime} t_{(2)}\right] .
$$

If we now apply the substitutions (4.18) with $\tilde{\kappa}=0$, then it is checked that Equations (5.8) and (5.9) yield the results (4.33) through (4.35), which proves the equivalence of the two approaches. 
It is a simple matter to show that the remarks made in $\S 3.4$ and at the end of the foregoing section concerning the possibility of the formation of shock fronts are still valid. In fact, we can make the following general comment. If we can assume that the coefficients that occur in the general Equation (2.15), being functions of invariants of stresses, are further analytic, their expansion in power series is always justified. The equations used in the above study may thus be taken to be the ones from these obtained by retaining terms up to quadratic in the stresses. It will follow from this that the growth equation for the amplitude for a model of a certain order and those for models of higher order will be the same as that we have obtained in §3.4. Such comments hold true also in classical hypoelasticity (cf. [29]).

\section{G. Concluding Remarks}

The above study has shown how important is the role played by initial stresses in the propagation of weak discontinuities and in the possible formation of shocks therefrom in relativistic elasticity. The importance of such results for the "seismology" of massive stellar objects in which a relativistic treatment is expected need not be emphasized. Of course, only the case of principal wave fronts can be studied analytically and it remains to achieve a thorough study of the hyperbolic character of the system, but computer calculations can be performed for more general cases. The possible occurence of shocks within a finite time interval however shows one of the limitation of the present study. If shocks occur, then it is likely that the condition (4.8) be no longer respected and that relatively large deformation processes take place. If this is the case, thermodynamics will play an essential role in the study. This means that the study of shock fronts must be based on the exact thermodynamical constitutive Equations (4.5) and (4.6). Furthermore, the strength of electromagnetic fields in the stellar objects of interest may endeavor the study of true magnetoelastic weak discontinuities and shocks on the basis of the equations derived elsewhere $[1,26,30]$. Finally, the coupling with gravitational discontinuities may then play a role. These prospects form the subject matter of other works.

\section{References}

1. Maugin, G. A. : Magnetized deformable media in general relativity. Ann. Inst. Henri Poincaré 15, 275-302 (1971)

2. Carter, B., Quintana, H.: Foundations of general relativistic high-pressure elasticity. Proc. Roy. Soc. Lond. A 331, 57--83 (1972)

3. Carter, B. : Elastic perturbation theory in general relativity and a variational principle for a rotating solid star. Commun. math. Phys. 30, 261--286 (1973)

4. Carter, B., Quintana, H. : Relativistic formulation of the neutron starquake theory. Ann. Phys. (N.Y.) 95, 74--89 (1975)

5. Maugin, G. A. : Harmonic oscillations of elastic continua and detection of gravitational waves. J. Gen. Relat. Gravitat. 4, 241--272 (1973)

6. Maugin,G. A.: On relativistic deformable solids and the detection of gravitational waves. J. Gen. Relat. Gravitat. 5, 13-23 (1974)

7. Synge,J.L.: A theory of elasticity in general relativity. Math. Zeit. 72, 82-87 (1959)

8. Pichon, G. : Théorèmes d'existence pour les équations des milieux élastiques. J. Math. Pures Appl. 45, $395-409$ (1966) 
9. Lamoureux,L.: Ondes asymptotiques et approchées en mécanique classique et relativiste. These Doct. ès Sci. Math., Univ: Paris (1974) [see especially, pp. 59-64]

10. Maugin, G.A.: Formulation des lois de comportement mécanique relativiste des milieux continus. Thèse Doct. ès Sci. Math., Univ. Paris (1975)

11. Lichnerowicz, A.: Ondes de choc, ondes infinitésimales, Rayons. In: Cattaneo,C. (Ed.): Relativistic fluid dynamics, pp. 87-204. (CIME, Bressanone, 1970). Rome: Cremonese 1971

12. Eringen, A.C., Suhubi,E. S.: Elastodynamics, Vol. I. New York: Academic Press 1974

13. Maugin, G. A.: Champ des déformations d'un milieu continu dans l'espace-temps de Minkowski. C.R. Acad. Sci. Paris 273 A, 65-68 (1971)

14. Lichnerowicz, A.: Théories relativistes de la gravitation et de l'électromagnétisme. Paris: Masson 1955

15. Maugin,G.A.: The principle of material frame indifference in general relativity. In: Ondes et radiations gravitationelles (Proc. Coll. Intern. C.N.R.S., Paris, 1973), pp. 331-338. Paris : C.N.R.S. (Ed.) 1974

16. Oldroyd,J.G. : Equations of state of continuous matter in general relativity. Proc. Roy. Soc. Lond. A 316, 1-28 (1970)

17. Eringen, A.C.: Mechanics of continua. New York: J. Wiley 1967

18. Truesdell,C., Noll,W.: Handbuch der Physik, Bd. III/3. Ed.: Flügge,S. Berlin, Heidelberg, New York: Springer 1965

19. Maugin,G. A.: Conditions de compatibilité pour une hypersurface singulière. Ann. Inst. Poincaré 24, 213-241 (1976)

20. Suhubi,E.S.: Thermoelastic solids. In: Eringen, A.C. (Ed.): Continuum physics, Vol. 2, pp. 173265. New York: Academic Press 1975

21. Carter, B.: Speed of sound in a high-pressure general relativistic solid. Phys. Rev. D 7, 1590-1593 (1973)

22. Truesdell,C.: General and exact theory of waves in finite elastic strain. Arch. Rat. Mech. Anal. 8, 263-296 (1961)

23. Fedorov, F.I.: Theory of elastic waves in crystals. New York: Plenum 1969 [translated from the Russian]

24. Whitham, G.B.: Linear and nonlinear waves. New York: Wiley-Interscience 1974

25. Maugin, G. A.: Etude des déformations d'un milieu continu employant la notion d'espace de Finsler. C.R. Acad. Sci. Paris 273 A, 474-476 (1971)

26. Maugin, G. A.: On the covariant equations of the relativistic electrodynamics of continua-III-elastic solids (to appear)

27. Murnaghan,F.D.: Finite deformation of an elastic solid. New York: J. Wiley 1951

28. Bland,D. R.: Nonlinear dynamic elasticity. Waltham: Blaisdell 1969

29. Nariboli, G. A.: The growth and propagation of waves in hypoelastic media. J. Math. Anal. Appl. 8, 57-65 (1964)

30. Maugin, G. A.: Relativistic theory of magnetoelastic interactions. I-III. J. Phys. (G.B.) A 5, 786802 (1972): J. Phys. (G.B.) A 6, 306--321 and 1647-1666 (1973)

Communicated by R. Geroch

Received November 11, 1976 